\title{
Correction to: Upper Campanian-Maastrichtian calcareous nannoplankton biostratigraphy and paleoecology in Wadi Qena, Eastern Desert, Egypt
}

\author{
Abdel Galil A. Hewaidy ${ }^{1} \cdot$ Madhat M. M. Mandur $^{2} \cdot$ Sherif Farouk $^{2} \cdot$ Ibrahim S. El Agroudy $^{2}$
}

Published online: 8 July 2019

(C) Saudi Society for Geosciences 2019

\section{Correction to: Arabian Journal of Geosciences (2019) 12:366} https://doi.org/10.1007/s12517-019-4485-y

The original version of this paper was published with error. Some of the figures are not added such as Plates 1-4 and represented by incorrect figures. Given in this article are the complete and correct figures and plates.

The online version of the original article can be found at https://doi.org/ 10.1007/s12517-019-4485-y

\footnotetext{
Ibrahim S. El Agroudy

ibrahim.esaaid@yahoo.com

1 Geology Department, Faculty of Science, Al-Azhar University, Nasr City, Cairo, Egypt

2 Exploration Department, Egyptian Petroleum Research Institute, 1 Ahmed El zomer, Nasr City, Cairo 11727, Egypt
} 
Fig. 1 Geological map of the study area showing the position of the Hawashiya and Umm Omeiyied sections, Eastern Desert, Egypt (modified Conoco 1987)

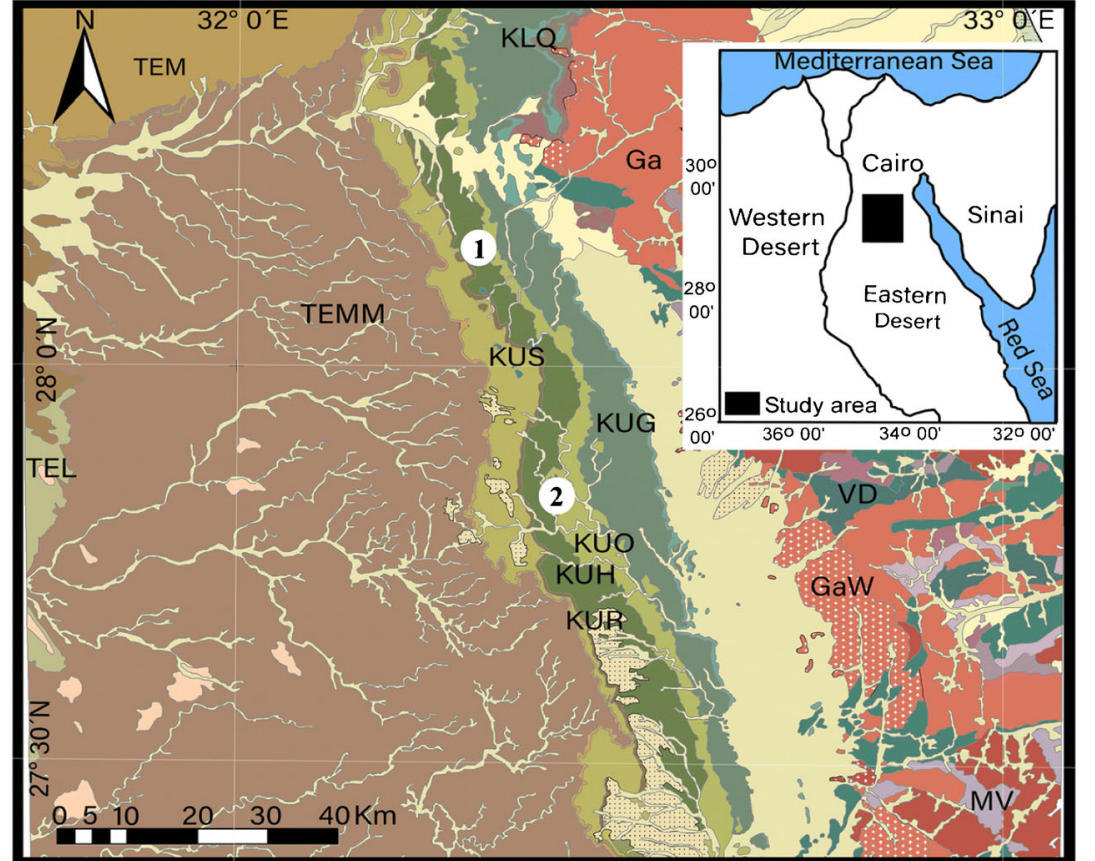

GaW Calc alkaline rock KLQWadi Qena Fm. KURRakhiyat Fm. TEMMMaghagha Fm. $\mathrm{Ga}$ Calc alkaline rock KUGGalala Fm. KUS Sudr Fm. 1. Hawashiya sections VD Dokhan volcanics KUOUmm Omeiyied Fm. TELMinia Fm. 2. Umm Omeiyied sec. MV Meta volcanics KUHHawashiya Fm. TEMMokkattam Fm. 


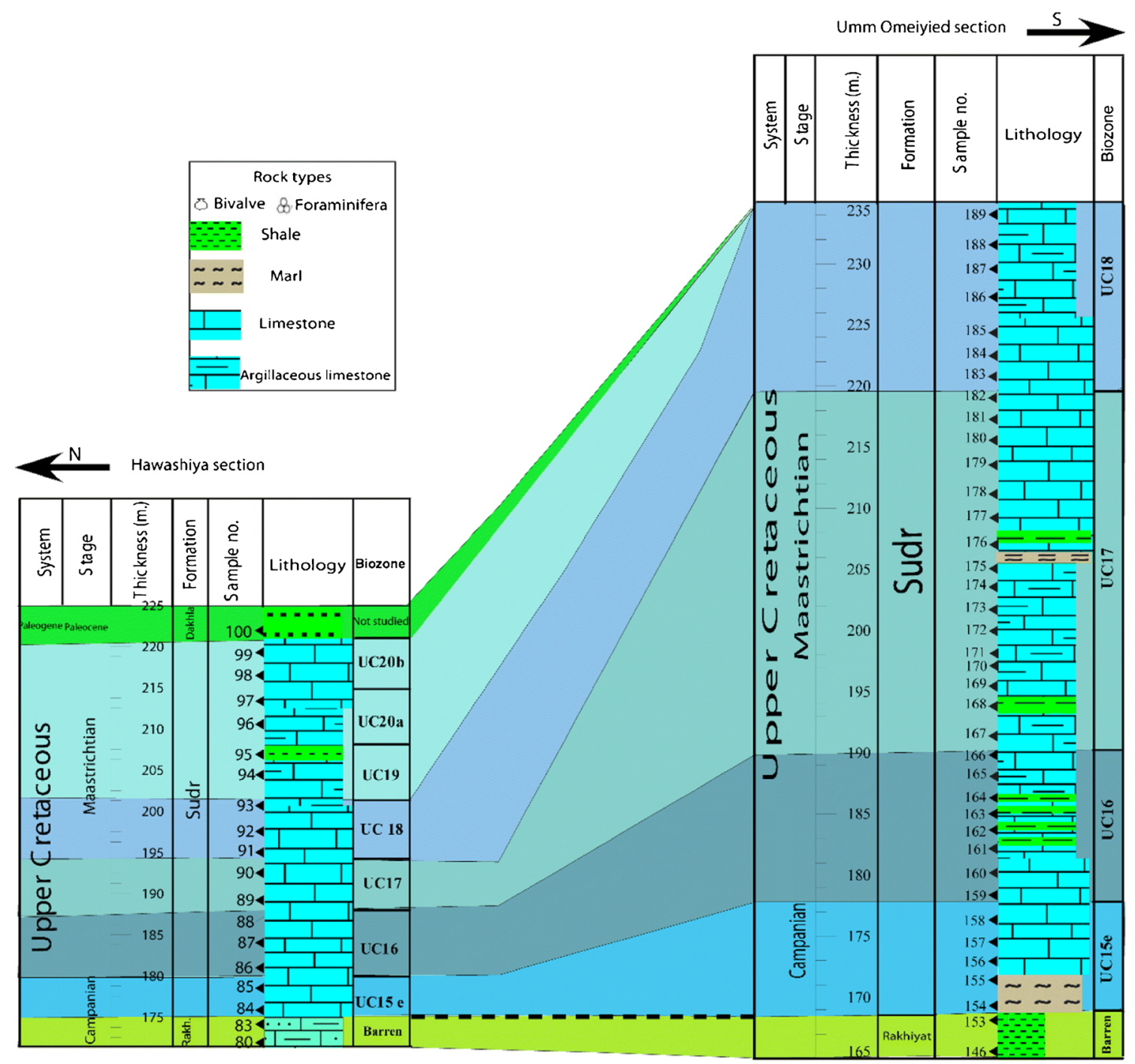

Fig. 2 Lithostratigraphic correlation chart of the Upper Campanian/Maastrichtian successions in the study area 


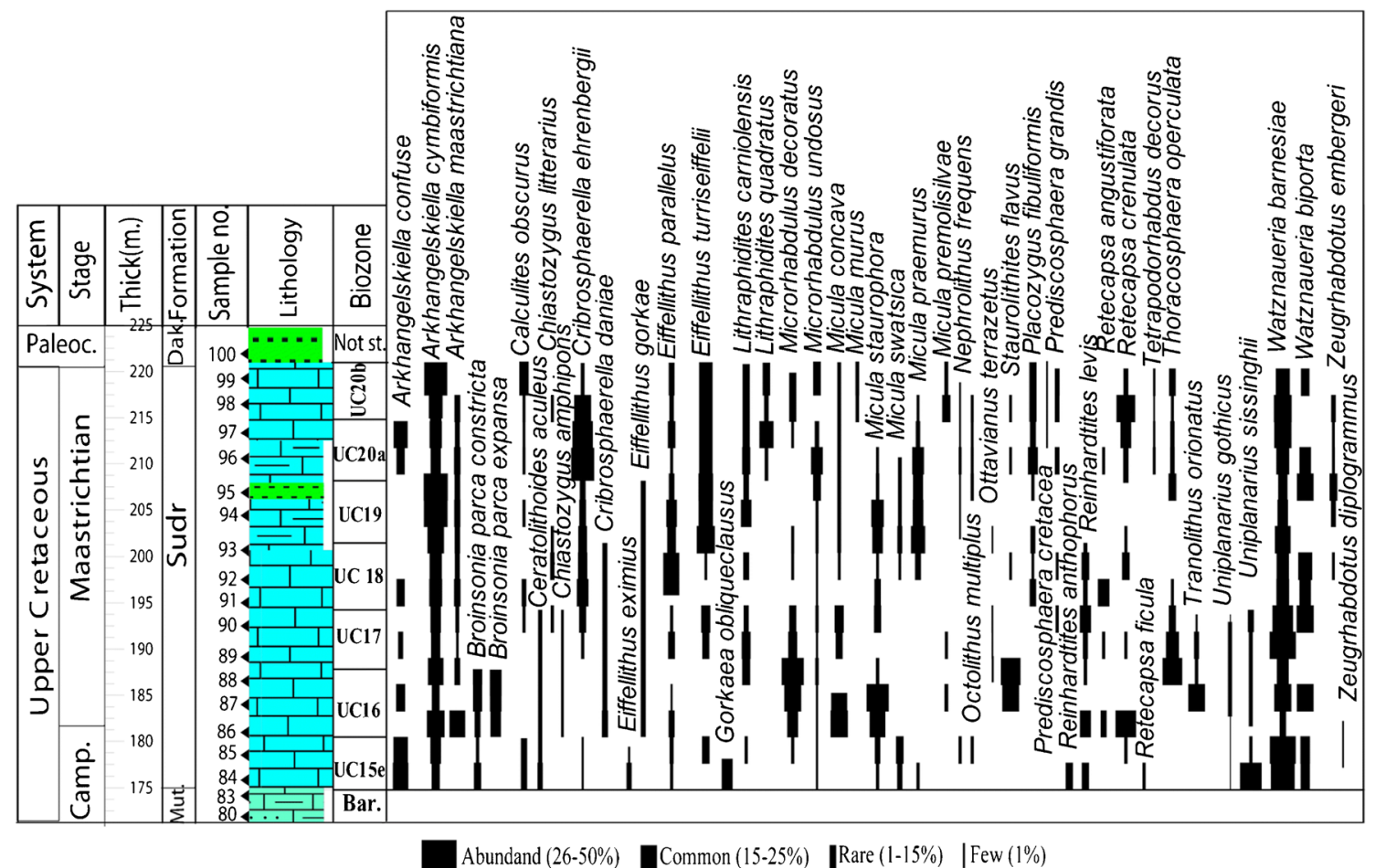

Fig. 3 Biostratigraphic distribution chart of the nannoplankton species recorded at Hawashiya section, Eastern Desert, Egypt

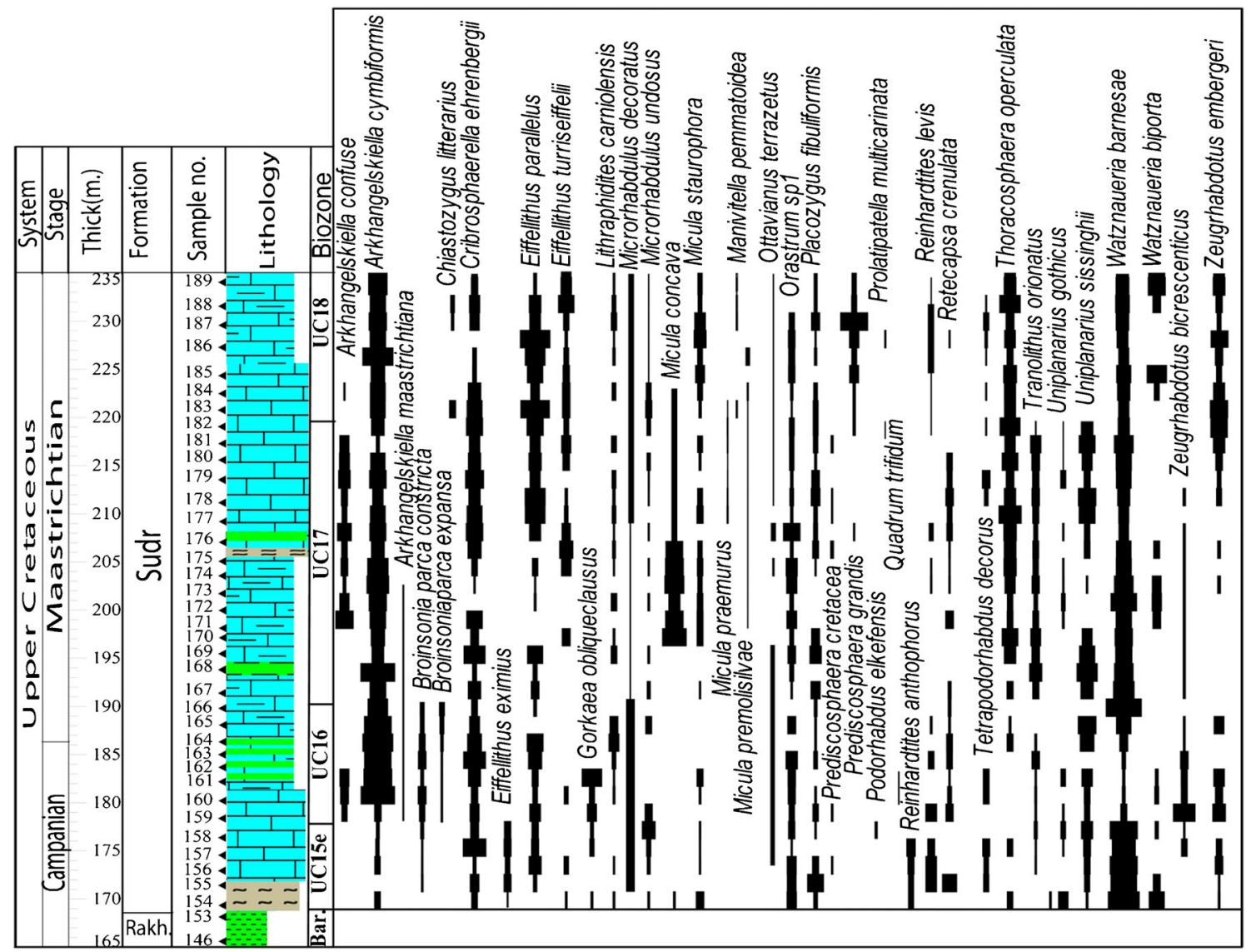

Fig. 4 Biostratigraphic distribution chart of the nannoplankton species recorded at the Umm Omeiyied section, Eastern Desert, Egypt 


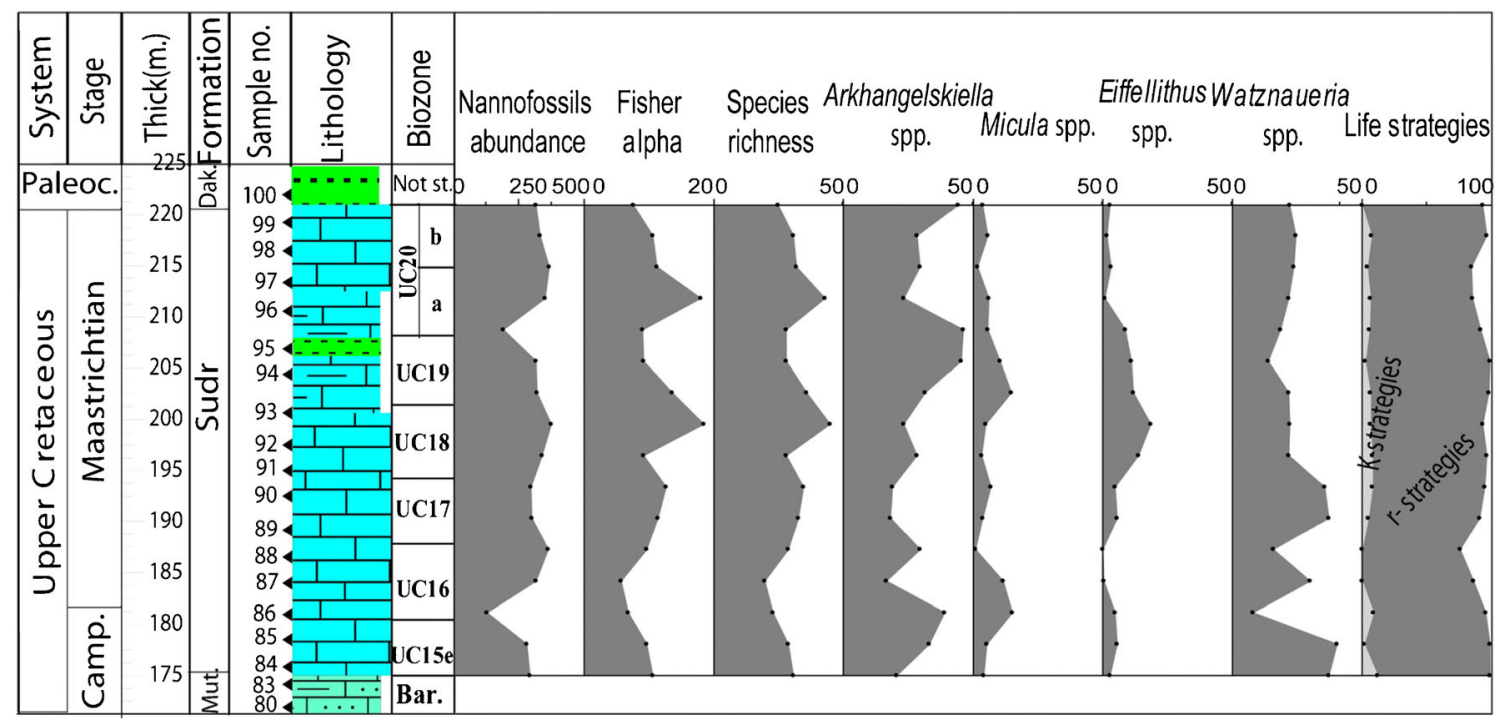

Fig. 5 Total nannofossil abundance, Fisher's alpha, species richness, relative abundances of most common nannofossil taxa in the Upper Eiffellithus spp., and Watznaueria spp.), and life strategies of Campanian-Maastrichtian (Arkhangelskiella spp., Micula spp.,

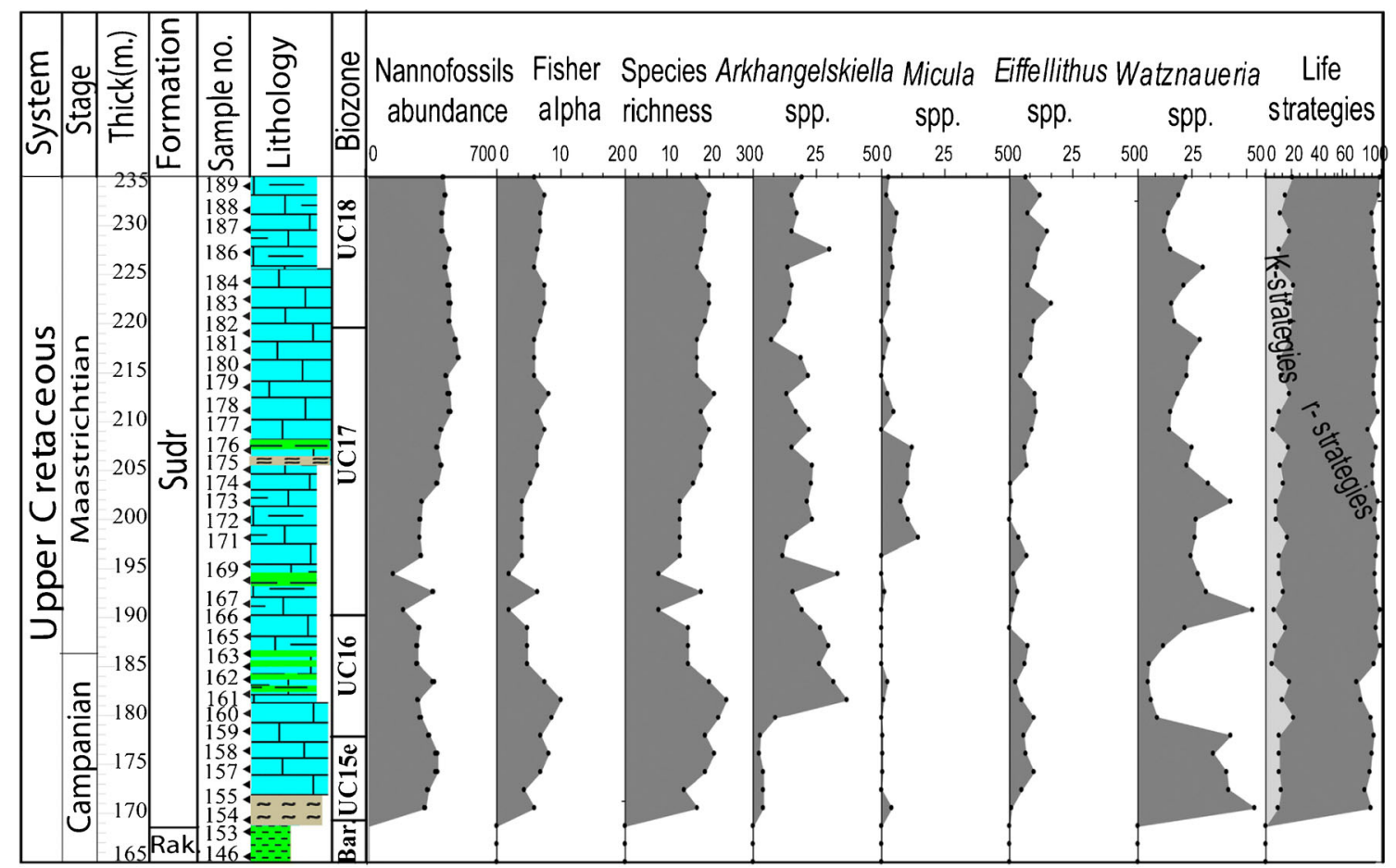

Fig. 6 Total nannofossil abundance, Fisher's alpha, species richness, relative abundances of most common nannofossil taxa in the Upper CampanianMaastrichtian (Arkhangelskiella spp., Micula spp., Eiffellithus spp., and Watznaueria spp.), and life strategies of Umm Omeiyied section 


\begin{tabular}{|c|c|}
\hline k-strategists & r-strategists \\
\hline $\begin{array}{l}\text { Calculites ovalis } \\
\text { Chiastozygus litterarius } \\
\text { Cylindralithus serratus } \\
\text { Micula concava } \\
\text { Manivitella pemmatoidea } \\
\text { Octolithus multiplus } \\
\text { Retecapsa ficula } \\
\text { Placozygus fibuliformis } \\
\text { Uniplanarius gothicus } \\
\text { Thoracosphaera operculata } \\
\text { Zeugrhabdotus embergeri } \\
\text { Zeugrhabdotu diplogrammus }\end{array}$ & $\begin{array}{l}\text { Arkhangelskiella cymbiformis } \\
\text { Arkhangelskiella confusa } \\
\text { Arkhangelskiella maastrichtiana } \\
\text { Broinsonia parca constricta } \\
\text { Calculites obscurus } \\
\text { Ceratolithoides aculeus } \\
\text { Cribrosphaerella daniae } \\
\text { Cribrosphaerella ehrenbergii } \\
\text { Eiffellithus gorkae } \\
\text { Eiffellithus eximius } \\
\text { Eiffellithus parallelus } \\
\text { Eiffellithus turriseiffelii } \\
\text { Lithraphidites carniolensis } \\
\text { Microrhabdulus decoratus } \\
\text { Micula swastica } \\
\text { Micula murus } \\
\text { Micula staurophora } \\
\text { Micula premolisilvae } \\
\text { Micula praemurus } \\
\text { Microrhabdulus undosus } \\
\text { Nephrolithus frequens } \\
\text { Prediscosphaera grandis } \\
\text { Relecapsa angustiforata } \\
\text { Relecapsa crenulata } \\
\text { Reinhardtites anthophorus } \\
\text { Reinhardtites levis } \\
\text { Tranolithus orionatus } \\
\text { Watznaueria biporta } \\
\text { Watznaueria barnesiae }\end{array}$ \\
\hline
\end{tabular}

Fig. 7 Frequency of nannofossils in respect to their ecological characteristics. The majority of individuals in the 2 - $\mu$ m fraction assign to opportunistic rstrategist taxa are represented by $80 \%$ each. And k-strategist taxa ranging between 0 and $20 \%$ (for methodology, see Lees 2002) 

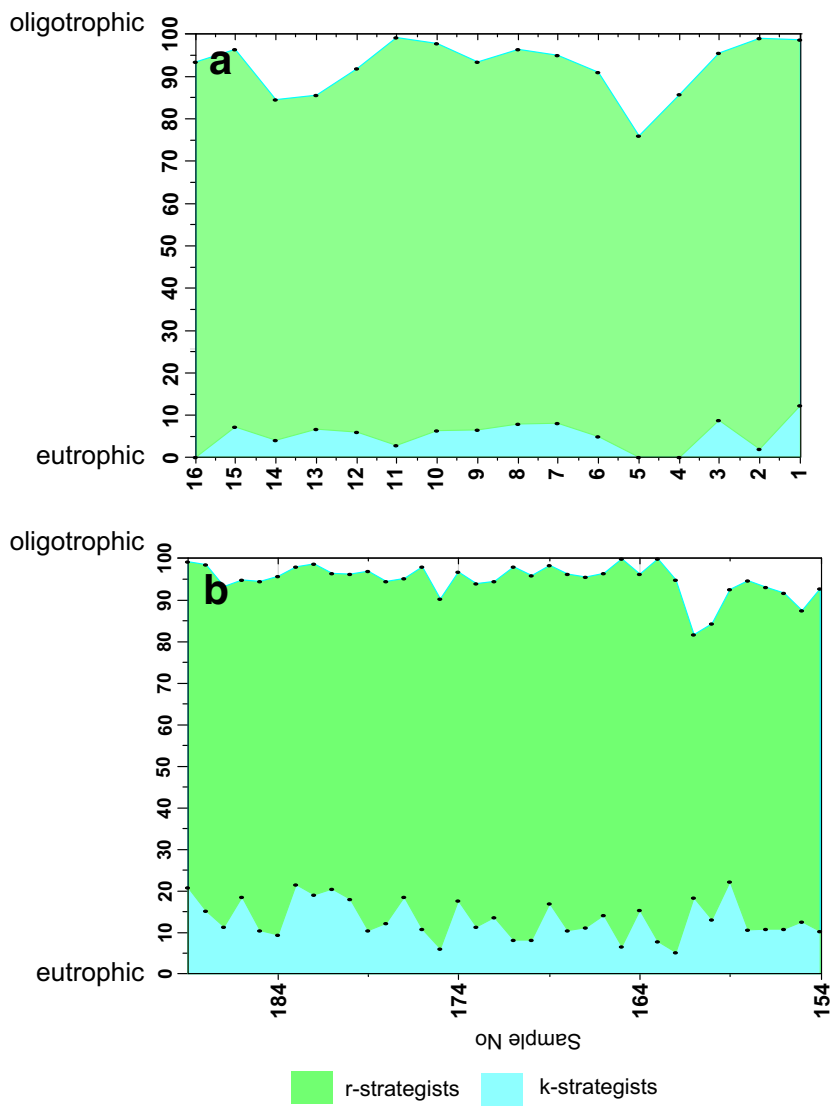

Fig. 8 a Life strategies of nannofossils at the Hawashiya section. b Life strategies of nannofossils at Umm Omeiyied section

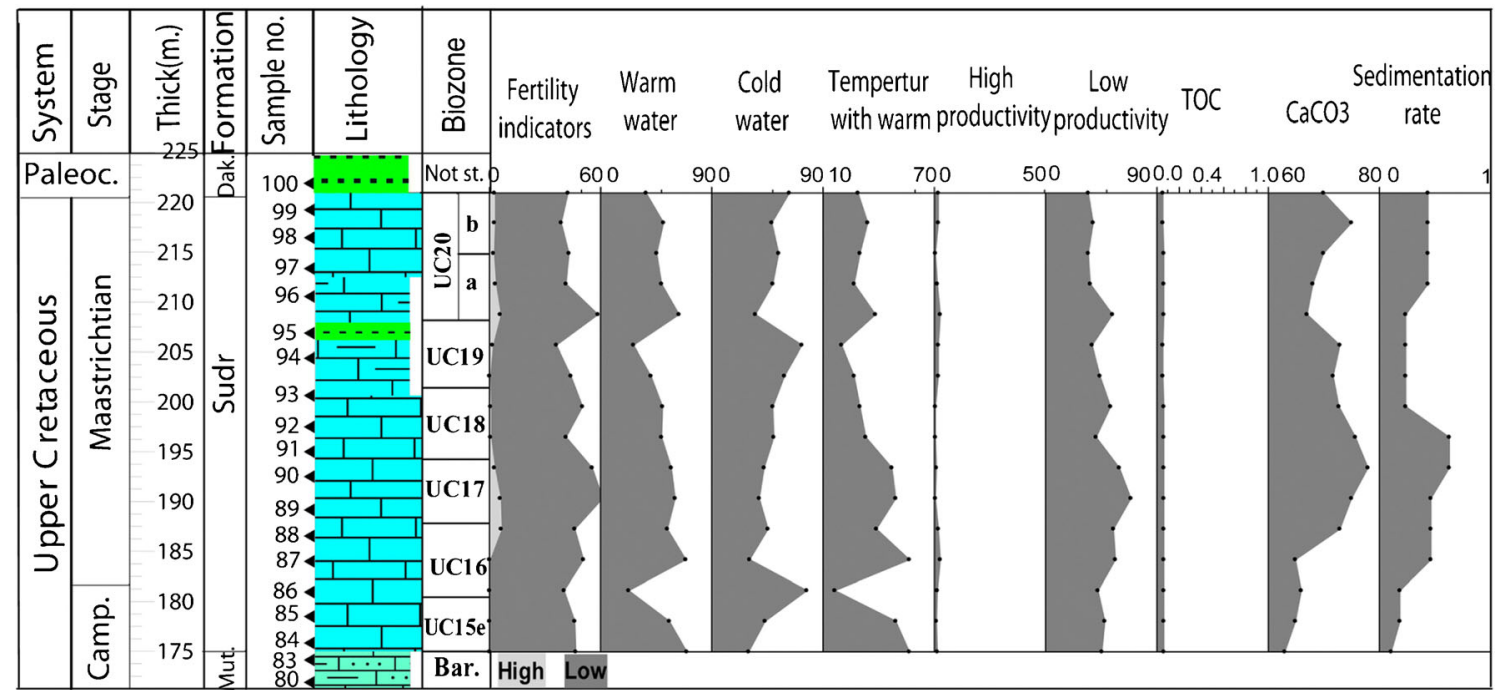

Fig. 9 Fertility indicators, warm water, cool water, temperature indication of warm, high productivity, low productivity, $\mathrm{TOC} \mathrm{CaCO}_{3}$, and sedimentation rate of the Hawashiya section 


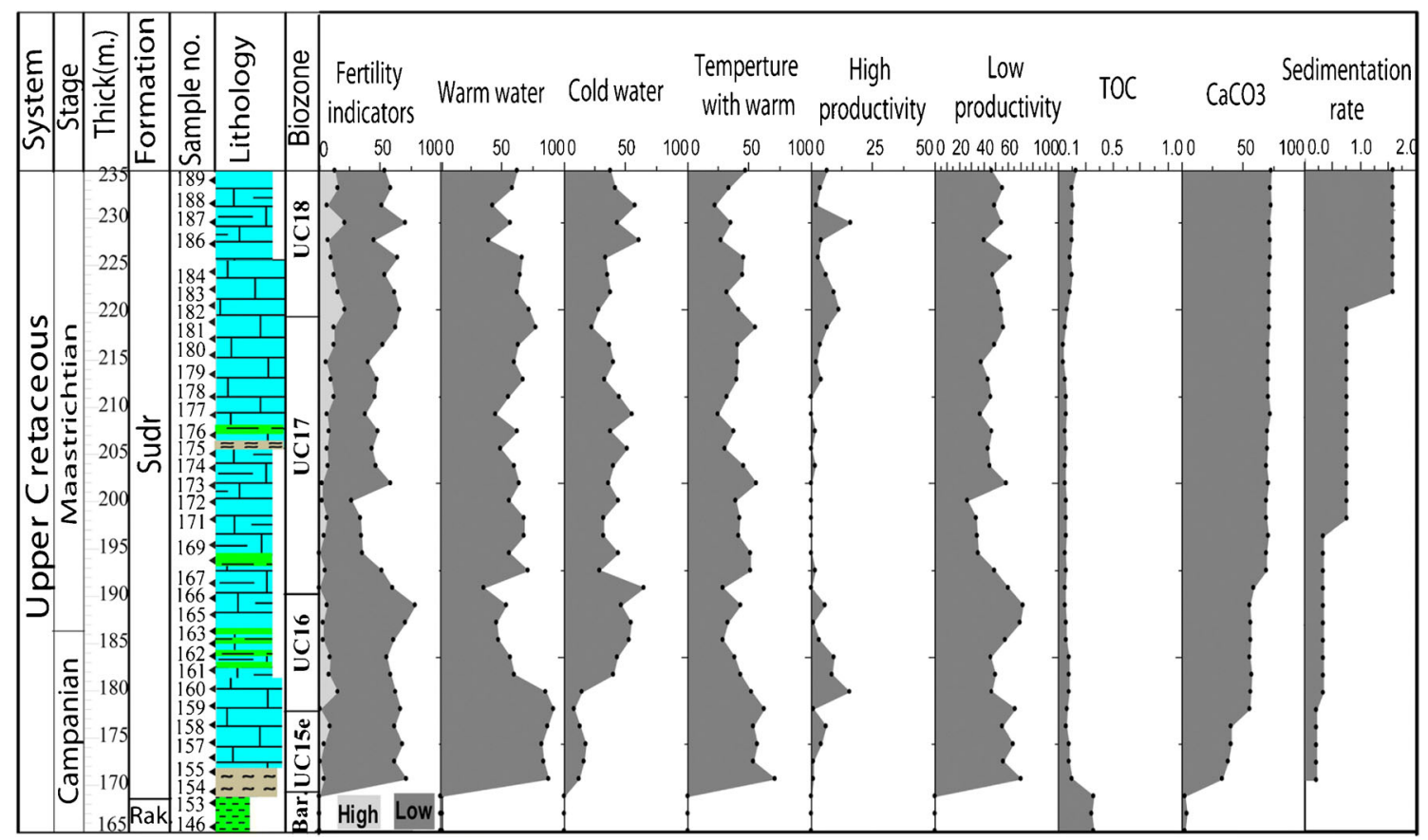

Fig. 10 Fertility indicators, warm water, cool water, temperature indication of warm, high productivity, low productivity, $\mathrm{TOC}, \mathrm{CaCO}_{3}$, and sedimentation rate of the Umm Omeiyied section

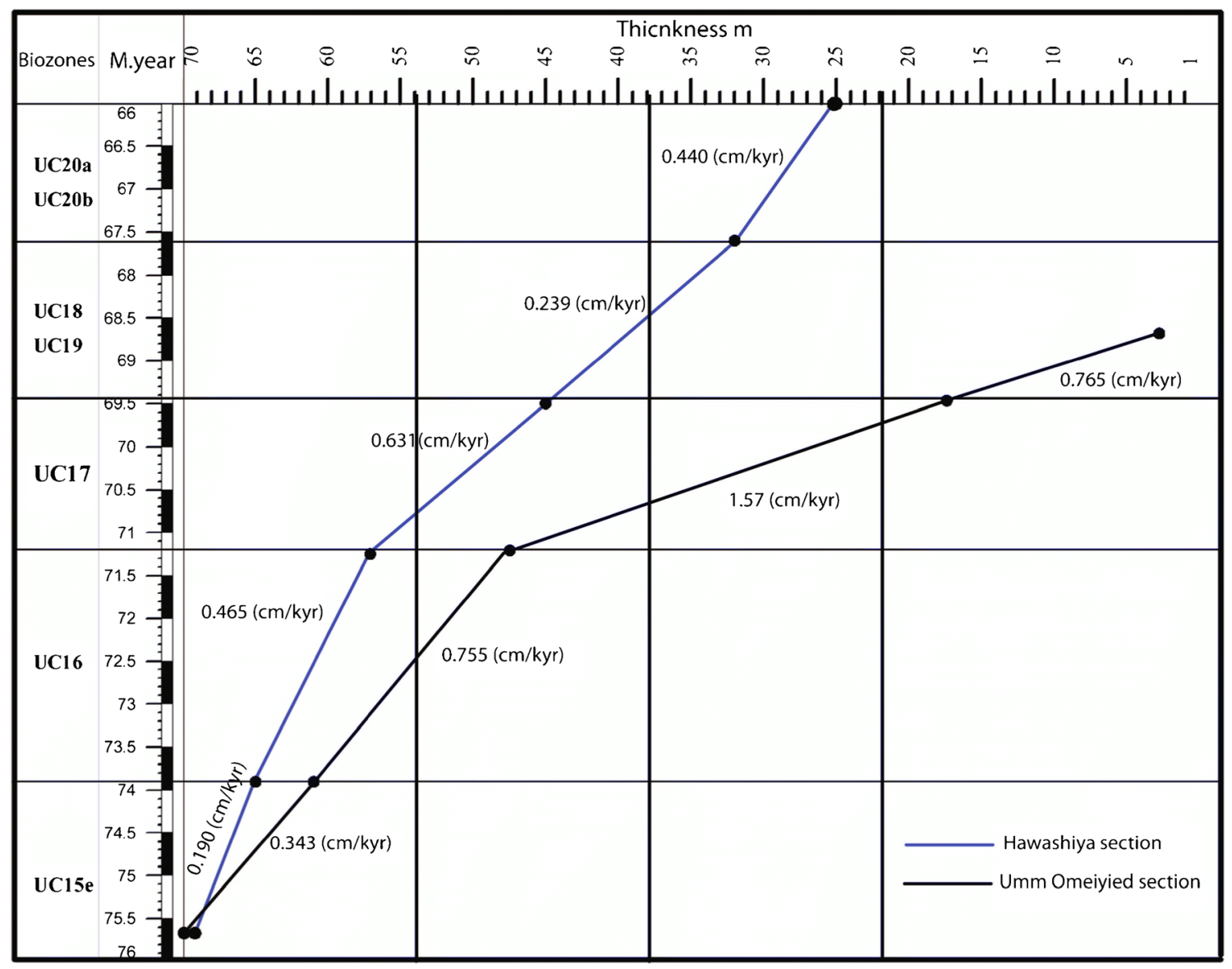

Fig. 11 Linear sedimentation rates calculated by calcareous nannoplankton datum age estimates and depths 

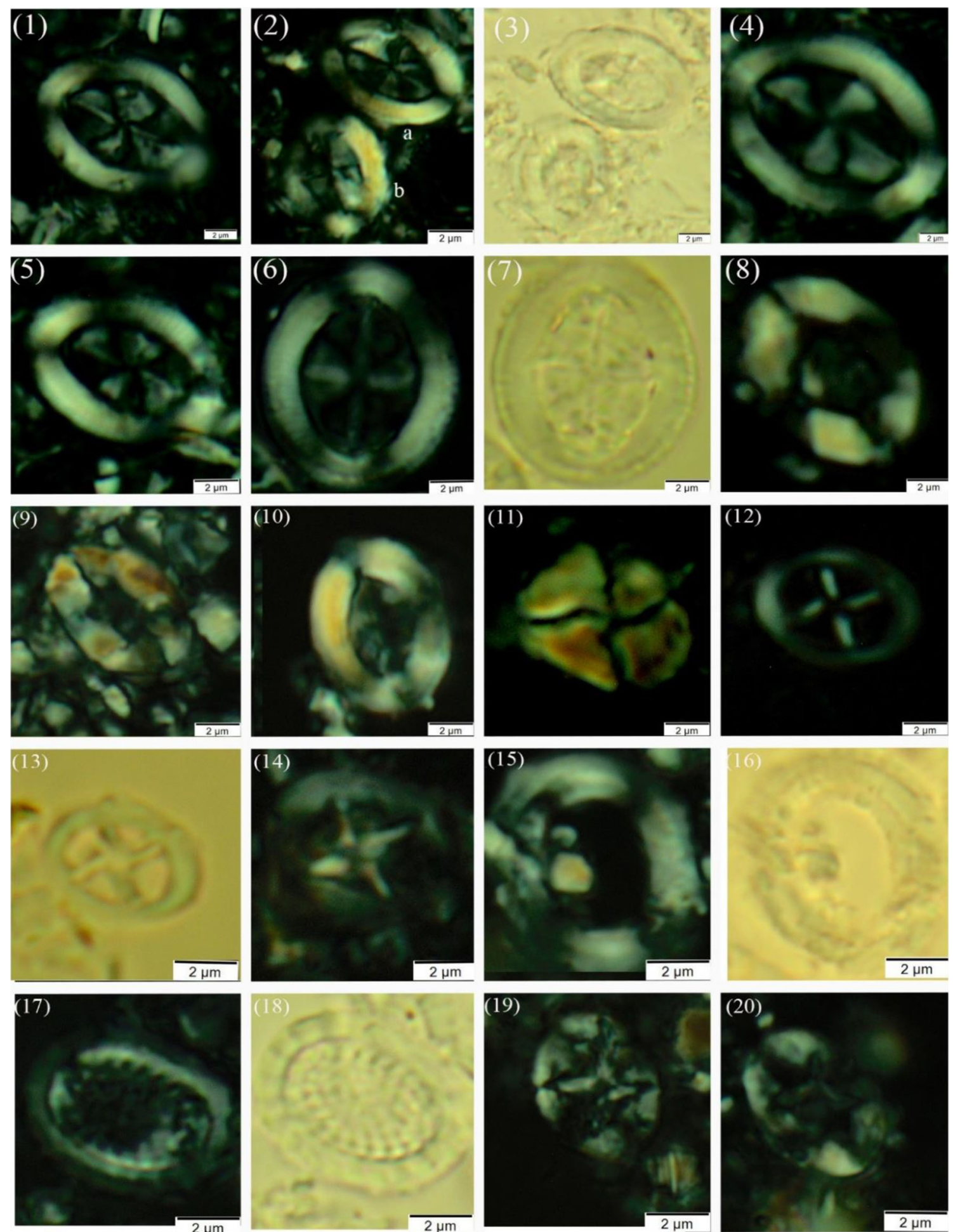

Plate 1 1-3 Arkhangelskiella confusa (Burnett 1998); sample 173, Sudr Formation, Umm Omeiyied section, Early Maastrichtian. 4-5 Arkhangelskiella cymbiformis Vekshina, 1959; sample 159, Sudr Formation, Umm Omeiyied section, Late Campanian. 6-7 Arkhangelskiella maastrichtiensis Burnett, 1997; sample 90, Sudr Formation, Hawashiya section, Early Maastrichtian. 8 Broinsonia parca constricta Hattner et al., 1980; sample 87, Sudr Formation, Hawashiya section, Early Maastrichtian. 9-10 Broinsonia parca subsp. expansa Wise \& Watkins in Wise (1983); sample 87, Sudr Formation, Hawashiya section, Early Maastrichtian. 11 Calculites obscurus (Deflandre, 1959); sample 92, Sudr Formation, Hawashiya section,

Early Maastrichtian. 12-13 Chiastozygus amphipons (Bramlette \& Martini, 1964); sample 90, Sudr Formation, Hawashiya section, Early Maastrichtian. 14 Chiastozygus litterarius (Górka, 1957); sample 90, Sudr Formation, Hawashiya section, Early Maastrichtian. 15-16 Cribrosphaerella daniae Perch-Nielsen, 1973; sample 93, Sudr Formation, Hawashiya section, Early Maastrichtian. 17-18 Cribrosphaerella ehrenbergii (Arkhangelsky, 1912); sample 185, Sudr Formation, Umm Omeiyied section, Early Maastrichtian. 19-20 Eiffellithus eximius (Stover, 1966), sample 157, Sudr Formation, Umm Omeiyied section, Late Campanian 

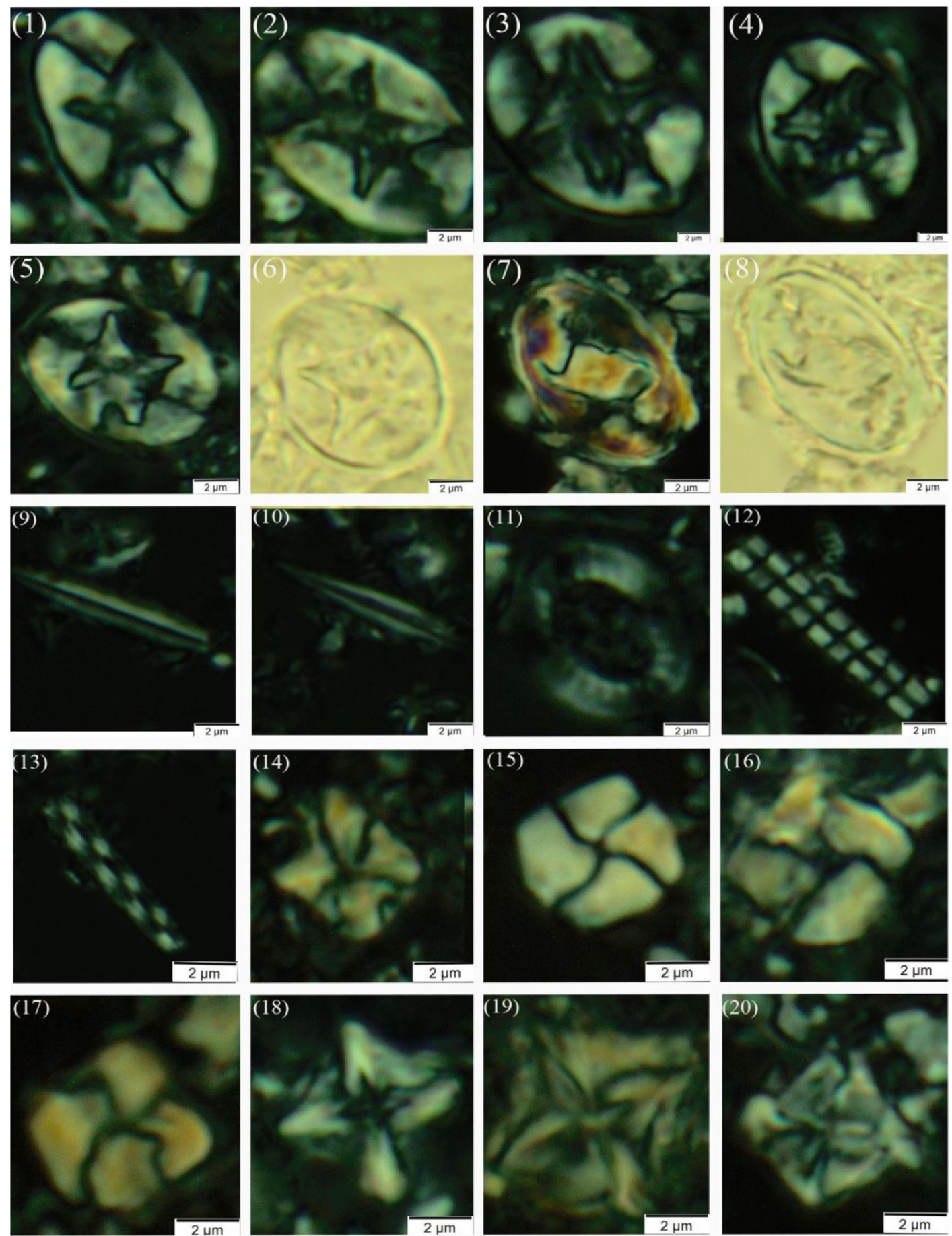

Plate 2 1-2. Eiffellithus gorkae Reinhardt (1965); sample 90, Sudr Formation, Hawashiya section, Early Maastrichtian3-4 Eiffellithus parallelus Perch-Nielsen (1973); sample 90, Sudr Formation, Hawashiya section, Early Maastrichtian5-6. Eiffellithus turriseiffelii (Deflandre in Deflandre \& Fert, 1954); sample 98, Sudr Formation, Hawashiya section, Late Maastrichtian7-8. Gorkaea obliqueclausus (Varol, 1991); sample 158, Sudr Formation, Umm Omeiyied section, Late Campanian.9. Lithraphidites carniolensis carniolensis Deflandre,1963; sample 181, Sudr Formation, Umm Omeiyied section, Early Maastrichtian.10. Lithraphidites quadratus Bramlette \& Martini, 1964; sample 97, Sudr Formation, Hawashiya section, Late Maastrichtian11. Manivitella pemmatoidea (Deflandre in Manivit, 1965), sample 98, Sudr Formation, Hawashiya section, Late Maastrichtian12. Microrhabdulus decoratus

Deflandre (1959) sample 88, Sudr Formation, Hawashiya section, Early Maastrichtian13. Microrhabdulus undosus Perch-Nielsen (1973), sample 157, Sudr Formation, Umm Omeiyied section, Late Campanian14. Micula concava (Stradner in Martini \& Stradner, 1960), sample 175, Sudr Formation, Umm Omeiyied section, Early Maastrichtian.15. Micula praemurus (Bukry, 1973), sample 178, Sudr Formation, Umm Omeiyied section, Early Maastrichtian.16. Micula premolisilvae Lees \& Bown, 2005; sample 186, Sudr Formation, Umm Omeiyied section, Early Maastrichtian.17. Micula murus (Martini, 1961); sample 99, Sudr Formation, Hawashiya section, Late Maastrichtian18. Micula staurophora (Gardet, 1955); sample 93, Sudr Formation, Hawashiya section, Early Maastrichtian. 19-20. Micula swastica Stradner \& Steinmetz (1984) sample 92, Sudr Formation, Hawashiya section, Early Maastrichtian 

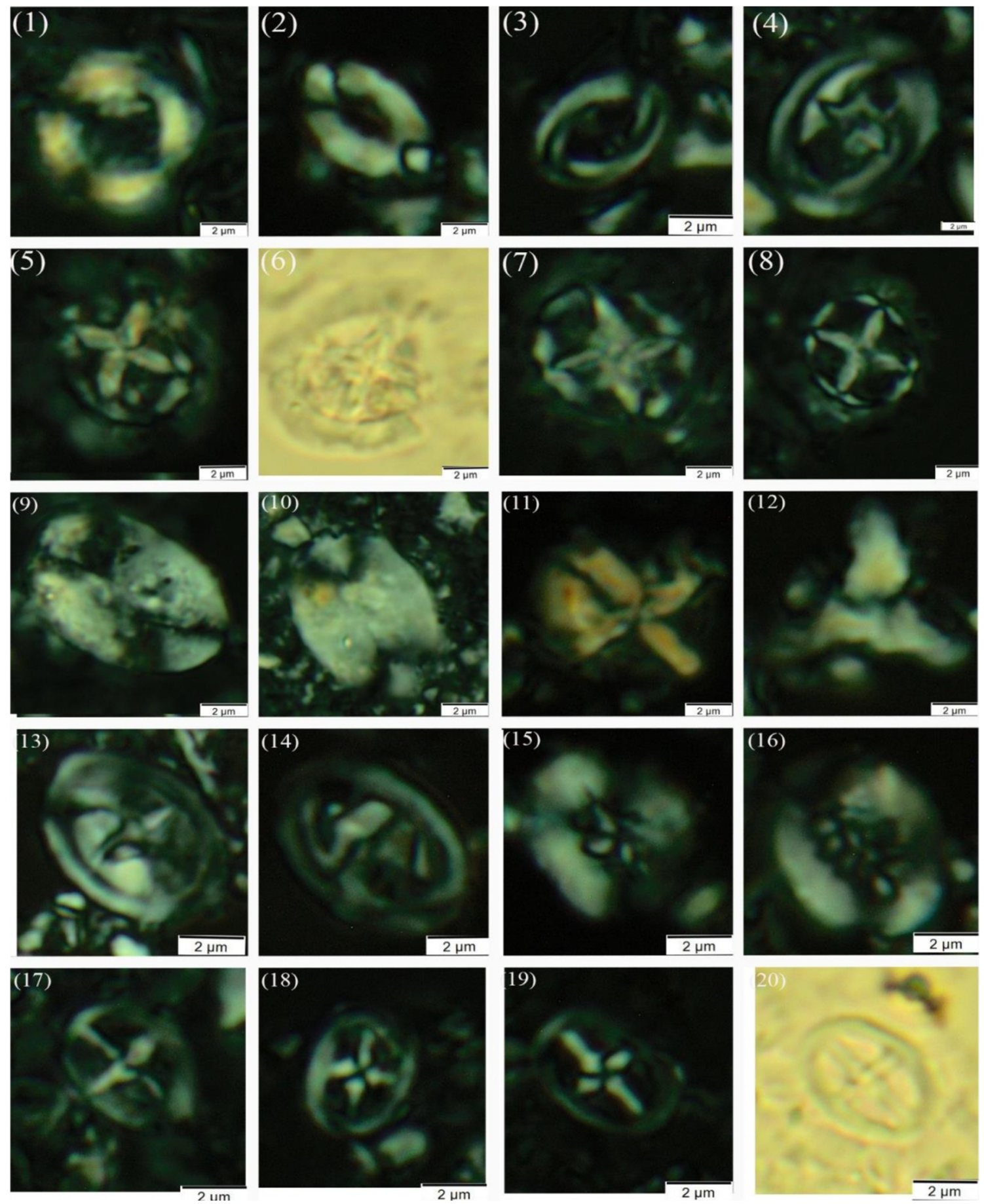

Plate 31 Orastrum spp. sample 97, Sudr Formation, Hawashiya section, Late Maastrichtian. 2 Ottavianus terrazetus Risatti, 1973; sample 92, Sudr Formation, Hawashiya section, Early Maastrichtian. 3-4 Placozygus fibuliformis (Reinhardt, 1964); sample 98, Sudr Formation, Hawashiya section, Late Maastrichtian. 5-6 Prediscosphaera cretacea (Arkhangclsky, 1912) sample 98, Sudr Formation, Hawashiya section, Late Maastrichtian. 7-8 Prediscosphaera grandis Perch-Nielsen (1979) sample 188, Sudr Formation, Umm Omeiyied section, Early Maastrichtian. 9-10 Prolatipatella multicarinata Gartner (1968)sample 186, Sudr Formation, Umm Omeiyied section, Early Maastrichtian. 11 Uniplanarius sissinghii Perch-Nielsen (1984b); sample 168, Sudr Formation, Umm Omeiyied section, Early Maastrichtian. 12 Uniplanarius trifidus (Stradner in Stradner \& Papp, 1961 sample 159,

Sudr Formation, Umm Omeiyied section, Late Campanian. 13 Reinhardtites anthophorus (Deflandre, 1959) sample 156, Sudr Formation, Umm Omeiyied section, Late Campanian. 14 Reinhardtites levis Prins \& Sissingh in Sissingh (1977) sample 188, Sudr Formation, UmmOmeiyied section, Early Maastrichtian. 15 Retecapsa angustiforata Black (1971) sample 96, Sudr Formation, Hawashiya section, Late Maastrichtian. 16 Retecapsa crenulata (Bramlette \& Martini, 1964); sample 98, Sudr Formation, Hawashiya section, Late Maastrichtian. 17 Staurolithites imbricatus (Gartner, 1968); sample 188, Sudr Formation, Umm Omeiyied section, Early Maastrichtian. 18-20 Staurolithites zoensis Burnett, 1997 sample 186, Sudr Formation, Umm Omeiyied section, Early Maastrichtian 

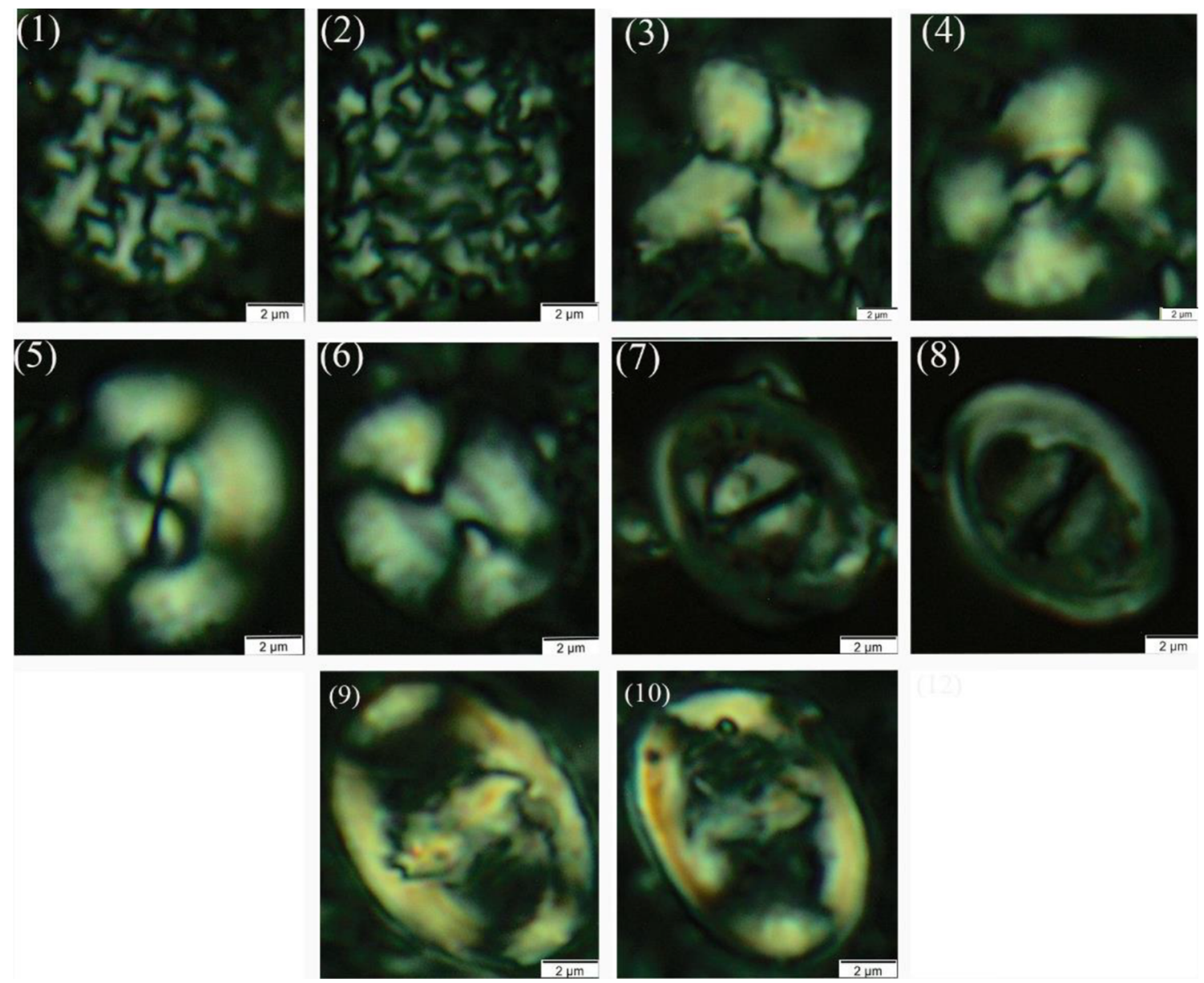

Plate 4 1-2 Thoracosphaera operculata Bramlette \& Martini (1964) sample 188, Sudr Formation, Umm Omeiyied section, Early Maastrichtian. 3 Uniplanarius gothicus (Deflandre, 1959); sample 179, Sudr Formation, Umm Omeiyied section, Early Maastrichtian. 4-5 Watznaueria barnesiae (Black in Black and Barnes 1959), sample 97, Sudr Formation, Hawashiya section, Late Maastrichtian. 6 Watznaueria biporta Bukry (1969), sample 98, Sudr Formation, Hawashiya section,

Late Maastrichtian. 7 Zeugrhabdotus bicrescenticus (Stover, 1966) sample 98, Sudr Formation, Hawashiya section, Late Maastrichtian. 8 Zeugrhabdotus diplogrammus (Deflandre in Deflandre \& Fert, 1954) sample 85, Sudr Formation, Hawashiya section, Early Maastrichtian. 910 Zeugrhabdotus embergeri (Noel, 1959); sample 98, Sudr Formation, Hawashiya section, Late Maastrichtian 